\title{
Management and Treatment of Type 1 And 2 Diabetes: State of Art
}

Tagliente $\mathbf{I}^{1,2,6^{*}}$, Digilio $\mathbf{G}^{2}$, Ullmann $\mathbf{N}^{3}$, Solvoll $\mathbf{T}^{5}$, Trieste $\mathbf{L}^{8}$, Murgia $\mathbf{F}^{1}$, Bella $\mathbf{S}^{7}$, Bini $\mathbf{F}^{2}$ and Schiaffini $\mathbf{R}^{4}$

${ }^{1}$ Scientific Direction, Health Care Innovations and Management, Bambino Gesù Children's Hospital, IRCCS, Rome, Italy

${ }^{2}$ Department of Information, Electronic and Telecommunication, University of Sapienza

${ }^{3}$ Respiratory Unit, Bambino Gesù Children's Hospital, IRCCS, Rome, Italy

${ }^{4}$ Unit of Endocrinology and Diabetes, Bambino Gesù Children's Hospital, Rome, Italy

${ }^{5}$ Norwegian Centre for Integrated Care and Telemedicine, University hospital of North Norway, Tromsø, Norway

${ }^{6}$ Department of Human Social and Health Sciences, University of Cassino and Southern Lazio, Cassino, Italy

${ }^{7}$ Department of Pediatric Medicine, High Specialization in Continuity of Care in Chronic Diseases, Pediatric Hospital Bambino Gesù, Rome, Italy

${ }^{8}$ Institute of Management, Scuola Superiore Sant'Anna, Pisa, Italy

"Corresponding author: Irene Tagliente, Respiratory Unit, Bambino Gesù Children's Hospital, Bambino Gesu Via Ferdinando Baldelli 41 00146, Rome, Italy, Tel: +39 066859 4760; Fax: +39 066859 4760; E-mail: irene.tagliente@opbg.net

Rec date: May 23, 2016 Acc date: June 28, 2016 Pub date: June 30, 2016

Copyright: ( 2016 Tagliente I, et al. This is an open-access article distributed under the terms of the Creative Commons Attribution License, which permits unrestricted use, distribution, and reproduction in any medium, provided the original author and source are credited.

\section{Abstract}

Background: Diabetes is a hormonal disorder that affects an estimated three hundred million people worldwide. In this review (meta-analysis) of the literature, we carefully analyzed the limits and methods of the management of diabetes in adolescents and adults.

Objective: The study focused on the identification of technologies and therapeutic procedures currently used and tried to identify possible factors for future improvement.

Methods: The literature review was carried out on search engines like: PubMed, ADA, Google Scholar and Google Patents. According to our search criteria, the selected articles were analyzed regarding evidence and critical issues reported by patients and clinicians. The results were then compared with the recalls of the U.S. Food and Drug Administration (FDA).

Results: The currently in use drugs and the insulin infusion systems vary a lot and simultaneous develop of new technology adds to existing chaos. This study revealed possible areas for improvements both from a technological and a medical point of view. For instance: the multiple causes related to the different timing of insulin absorption according to the insertion site, the design of new algorithms focused on simplification of practical activities, and the identification of new methods for training patients and families on the different device's functions. Moreover, some critical points were emerged related to the current limitations and lack of interchangeability between sensors for monitoring vital parameters. This condition restricts the correct sensor selection according to the patient's personal preference, age and needs. However, the lack of interchangeability between different devices limits the choice of the most appropriate monitoring software and data storage.

Conclusions: The collaboration between medical doctors and biomedical engineers is of great importance when analyzing available medical devices for the purpose, identifies possible weaknesses, technical problems, and areas for improvements. Moreover, multi-specialist collaboration is important to conceive new methods of training for patients and families.

Keywords: Diabetes type 1; Infusion pump; Technical problems; European norms; Medical devices; Self-management; CGM; Stylus injectors; Glucometers; Software management

\section{Abbreviations}

CGM: Glucose Monitoring In Continuous; CG-EGA: Continuous Glucose-Error Grid Analysis; CSII: Continuous Subcutaneous Insulin Infusion; DM1: Diabetes type 1; DM2: Diabetes type 2; EGA: Error Grid Analysis; IFCC: International Federation of Clinical Chemistry and Laboratory Medicine; MARD: Median Absolute Relative Difference; NIST: National Institute of Standards and Technology

\section{Introduction}

Diabetes is a hormonal disorder that affects around three hundred million people worldwide [1]. Several therapies have been proposed and a good compliance to treatment offers patients a good quality of life. Diabetes is a disease characterized by a group of metabolic disorders caused by defects of insulin's secretion and / or activity. The first condition is represented by a pancreas inability to produce insulin and the latter is characterized by incorrect use of normal secreted insulin (insulin resistance). Produced in the beta cells from the Langerhans islets, insulin has a dual function: to transport glucose from the bloodstream to the body's cells, where it is converted into energy and it is involved in the anabolic metabolism of glycogen. 
Glucose, produced by the digestion of food, is transported by the blood to all body cells. The rate of glucose in the blood is called glycemia. Insulin is essential to allow the cells to use glucose as an energy source. Diabetes is divided into mellitus TYPE1 and TYPE2:

Diabetes type 1 (DM1) is characterized by immune-mediated destruction of pancreatic beta cells with absolute insulin deficiency. This condition needs to be treated with insulin injections or administered through a pump. DM1 appears more frequently in children and adolescents and accounts for about $5 \%-10 \%$ of all cases of diabetes in the world.

Diabetes type 2 (DM2) can be characterized by two conditions: inadequate insulin's secretions or peripheral insulin resistance. This is by far the most common form of this disease, with $90 \%$ - 95\% of cases. DM2 usually can be controlled with diet, physical activity and oral drugs but, in a high percentage of patients, insulin administration via syringes or infusion pumps are needed. Several clinical trials show that obesity and being overweight can cause the onset of type 2 diabetes.

In this paper, we present a review of the literature about the limits and methods of management of diabetes in adolescents and adults. The goal of the study was identify technologies and therapeutic procedures currently used for diabetes' treatment and monitoring and subsequently, highlighting possible areas for improvement. The aim was analyzing challenges and limits of currently available medical devices for the management of diabetes. The focus has been extended to stylus injectors, insulin pumps, sensors for Glucose Monitoring in Continuous (CGM), glucometers and finally the artificial pancreas. Therefore, in this paper we collected the European regulations for manufactures. The study highlighted shows possible ideas for both technological and scientific improvement.

\section{Methods}

\section{Research setting}

The study was conducted at Bambino Gesu' Children's Hospital, Rome Italy. The Hospital encourages healthcare providers to establish an open and friendly communication relationship with patients and families. Moreover, healthcare providers are recommended to provide accurate information about medical conditions and treatments, that can help patients and families deal with the illness in a more informed manner.

\section{Research method}

The literature review was carried out via the major search engines (PubMed, ADA, Google Scholar, Google Patents, CDA) considering the following keywords: diabetes, insulin pump, glucometer, biosensor, pen injector, oral hypoglycemic agents, insulin therapy, pancreas artificial, closed-loop, CGM, continuous Subcutaneous Insulin Infusion (CSII). From the first analysis ( ${ }^{\circ} 2000$ Articles) were selected abstracts and relevant full text of interest. The research led to the selection of the 15 items listed in the references. From the articles selected were analyzed evidence and critical issues reported by patients and clinicians, exposing the current technological advances aimed at overcoming those limits (bio-artificial pancreas, nano-biosensors, closed loop system). The selection of publications was performed according to: number of citations, year of publication (2004-2013), IF of the journal, significance of the results and published data the authority of the authors (we chose to consider only items written by diabetologists who strongly contributed to the scientific community taking part of National or International commission and or authors of guideline or medical books) and, finally, the importance of the conference if the article was presented. Finally, we defined the role that biomedical engineer must have to introduce these improvements in therapy for the treatment of diabetes.

Emergence was compared with the recall of the U.S. Food and Drug Administration (FDA) to the manufacturers of medical devices as glucometer or insulin pumps and deepened through the information made available by manufacturers.

Therefore in this paper we collected the European regulations for manufactures.

\section{Data Analysis}

\section{Stylus injectors}

The pen, or pen needle, is very practical and convenient for easy to control diabetes. They can be recharged or disposable and are visually similar to a pen. In the case of rechargeable Stylus injectors, a reservoir containing a vial of insulin (vial format pencil), whose apex is present with a wheel which can be set and modified from time to time, representing the doses needed for the patient. On one end there is a button to release insulin while at the opposite side (tip) there is the needle.

The advantages found by clinicians and users are: the ability to set the amount of insulin with extreme simplicity (it is set to a numeric selector), the needle is disposable and the dimensions of the stylus injectors are reduced in order to make discreet its use. The main disadvantage is that the units are not divisible, therefore it's not possible to use fractional units and this seriously limits treatment's adaptation for patients. In addition, when different types of insulin are needed, more stylus injectors would be also needed [2-5].

\section{Insulin pumps}

The insulin pump is a small precision electro-mechanical device that releases insulin into the body via a thin plastic tube (or infusion set).

The infuser is a long thin plastic tube (thin plastic tubing), which connects the pump to a small needle or flexible cannula, inserted through the skin at the point of infusion (infusion site), usually in the abdominal area in contact with the interstitial fluid. The infuser can be held under the skin for about three days, after which it must be replaced to avoid problems of infection. Unfortunately, the pump is not able to self-define the correct insulin dosage based on the blood glucose measurements. The pump is a precise instrument controlled by a small programmable computer, which releases insulin in previously set dosage (CSII) according to diabetologist prescription [6]. The pump can be programmed to release the drug at any time. However, the patient must check the blood glucose levels several times a day, a factor that, in parallel, requires much more attention by the patient compared to traditional systems of injection [6]. Some Insulin pumps are selflock in case of hypoglycemia thanks to special sensors [7].

\section{Sensors for glucose monitoring in continuous (CGM)}

A system for continuous measurement of blood glucose consists of a subcutaneous sensor which detects the concentration of glucose in the interstitial fluid. This system is connected to a transmitter that sends 
Citation: Tagliente I, Digilio G, Ullmann N, Solvoll T, Trieste L, et al. (2016) Management and Treatment of Type 1 And 2 Diabetes: State of Art. Gen Med (Los Angeles) 4: 259. doi:10.4172/2327-5146.1000259

Page 3 of 5

data to a receiver handheld which processes all information in order to adjust the insulin infusion (by the patient). Technological advances have increased precision and accuracy of analytical biosensors. The sensors currently available can reach a maximum MARD (Median Absolute Relative Difference), including measurement of blood glucose and blood glucose sensor of reference, between $11 \%$ to $14 \%$ [7]. Crucial indicator for the prescription of an effective therapy, but nevertheless it cannot be considered as an absolute indicator [8]. The MARD, as the Continuous Glucose-Error Grid Analysis (CG-EGA), is a Windows-based, statistical program designed to be used by the diabetes technology industry for the evaluation of continuous glucose monitoring devices. It is a logical extension of the original error grid analysis (EGA), which was developed to assess the clinical accuracy of patient-determined blood glucose values using either estimation or self-monitoring blood glucose systems. CG-EGA is based on the idea that information, generated by a monitoring system, should be reliable enough to result in clinically accurate decision. The CG-EGA technology was originally developed by Drs. William Clarke and Boris Kovatchev at the University of Virginia in Charlottesville, VA, USA.

Glucometers: A portable medical device is able to estimate with good approximation the glucose concentration in the blood. To estimate the blood glucose, the glucometer analyzes a small drop $(1 \mu \mathrm{L})$ of blood - usually taken from fingertip - on a special test strip. In most of the models, the glucose content in the blood sample activates an enzymatic reaction oxidizes by the presence of the enzyme glucose oxidase in the test strip; this oxidation produces a color change or an electrical current (depending on the model) convertible to glucose value [8].

\section{Software Management}

The main goal is to facilitate the control of the variance as a function of glycemic carbohydrate ingested and insulin units needed. The currently available devices, interfaced with PC, have their software for the storage and consultation of recorded data, allowing the visualization of the parameters recorded for more than 24 hours [9].

\section{European Norms}

The European regulations that manufacturers must follow are (from the most recent to the most dated):

UNI EN ISO 15197:2013: Systems of in vitro diagnostic assay Requirements for systems for self-testing of blood glucose monitoring in the treatment of diabetes mellitus.

Directive EN 61326-1:2006: Electrical equipment for measurement, control and laboratory use - EMC requirements - Part 1: General requirements.

Directive EEC / EAEC / EC n ${ }^{\circ} 79$ of 27/10/1998: 98/79/EC: Directive of the European Parliament and of the Council of 27 October 1998 on in vitro diagnostic medical devices.

Directive 1999/5/EC of the European Parliament and of the Council of 9 March 1999 on radio equipment and telecommunications terminal equipment and the mutual recognition of their conformity.

Directive 93/42 EEC for Medical Devices (abbreviated as DDM 93/42).

Reference Methods for the execution of medical devices validation tests:
Standard of the National Institute of Standards and Technology (NIST).

Standards and Recommendations of the 'International Federation of Clinical Chemistry and Laboratory Medicine (IFCC)'.

\section{Recall}

After this first phase we checked the major recall of FDA to the manufacturer of devices (Figure 1), numbers of recalled (Figure 2).

Therefore, we analyzed the why of the recalled to understand if there were some common factors to be studied, to perform the management of diabetes with devices (Figure 3).

The analysis was principally focused on glucometer and insulin pump. We studied the way of recalls, the motivation (e.g., was analyzed also the hardware that caused the error) and the risk for the patients as showed by diagrams on (Figure 4).

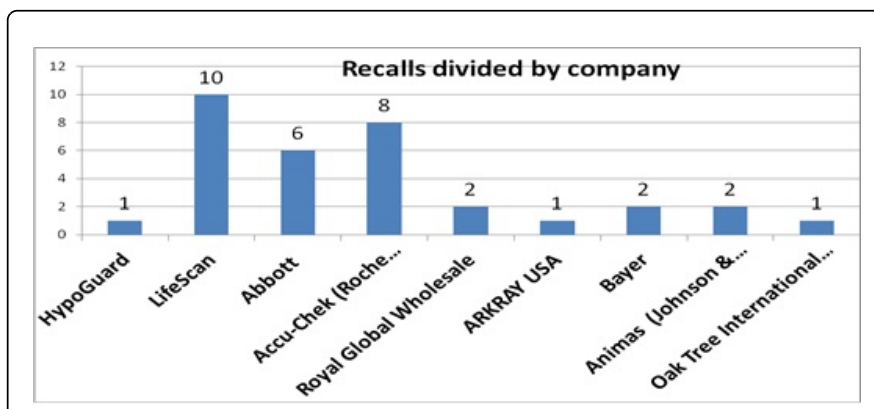

Figure 1: Number of recall for each manufacturer.

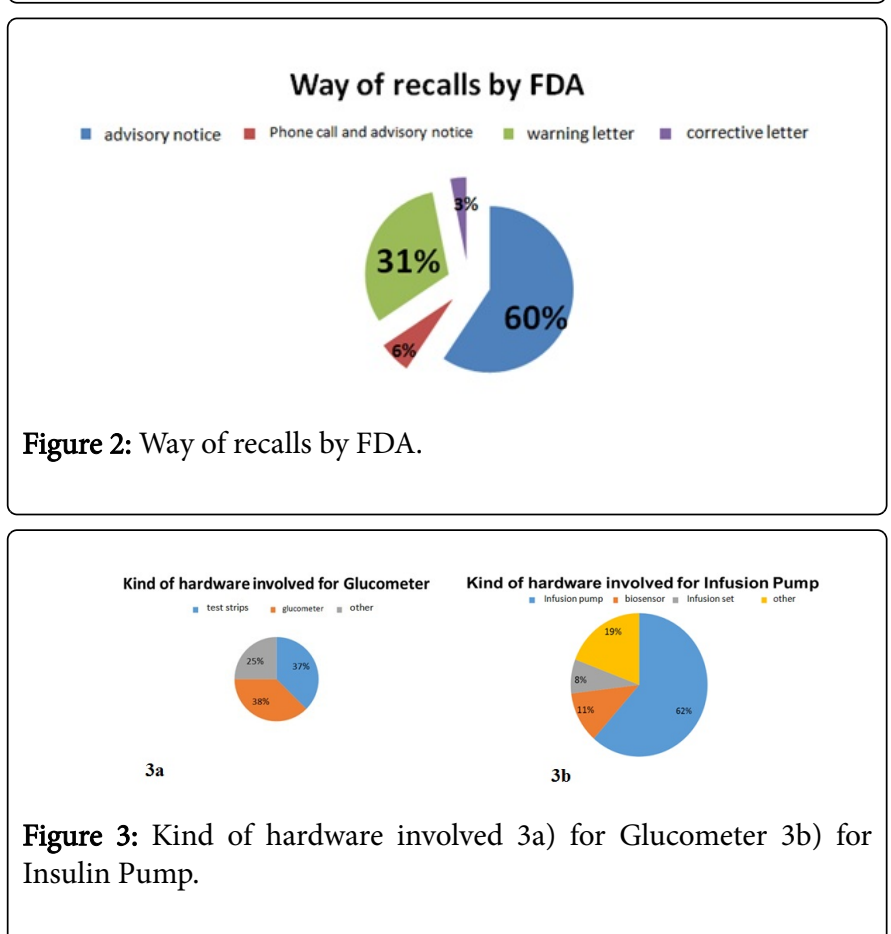




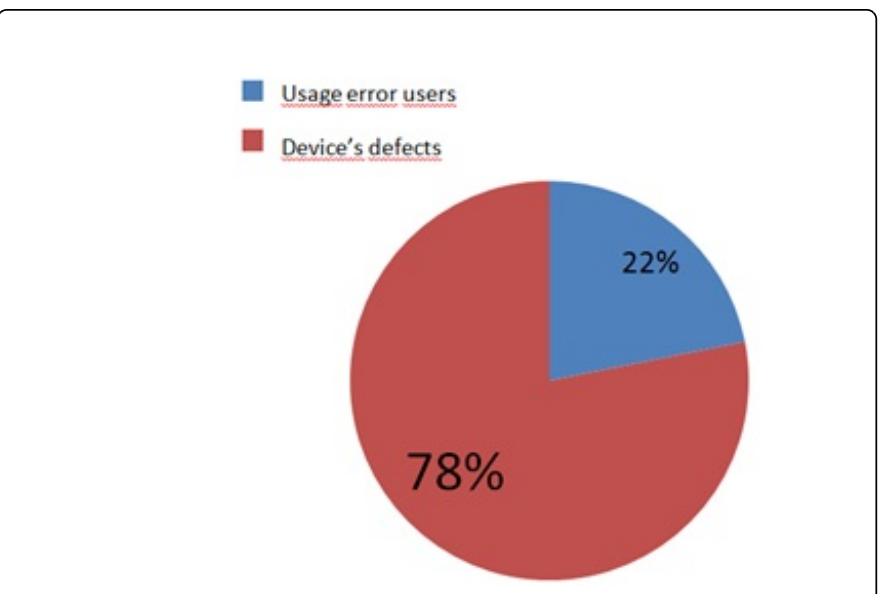

Figure 4: Alert percentage divided by type of device's defects or user's error.

\section{Results}

The intent of this study was to identify the state of art of the limits and methods of management of diabetes type 1 and 2 through the literature analysis.

Various are the drugs and the insulin infusion systems currently in use and under development [10].

Great is the industry attention to miniaturize all devices, with the use of nanotechnology, as well as to study biomaterials that are more suitable for insulin infusion.

Among many topics covered in the literature (Table 1), there are three issues considered of greater impact in the short-medium term.

\begin{tabular}{|l|l|}
\hline Factors & Desired improvements \\
\hline Insulin administration & $\begin{array}{l}\text { Identification of new techniques to } \\
\text { increase the rate of absorption of } \\
\text { insulin (ultra rapid wording, dosing in } \\
\text { other compartment, warmth the } \\
\text { infusion site) }\end{array}$ \\
\hline Training & $\begin{array}{l}\text { Identify new methods and training for } \\
\text { patients and their families to facilitate } \\
\text { the use of all the functions of the } \\
\text { various devices that can be used for } \\
\text { the monitoring and treatment of } \\
\text { diabetes type I and II }\end{array}$ \\
\hline $\begin{array}{l}\text { Management } \\
\text { communication protocols }\end{array}$ & $\begin{array}{l}\text { Example: 1) Communication between } \\
\text { glucose sensor and insulin pump: } \\
\text { make the connections more reliable; 2) } \\
\text { Interoperability of devices and } \\
\text { interfacing software: standardization of } \\
\text { the communication protocol }\end{array}$ \\
\hline $\begin{array}{l}\text { Improving the performance of the } \\
\text { algorithms }\end{array}$ & $\begin{array}{l}\text { Example: 1) Recognition of glucose: } \\
\text { Reduce the false positive alarms of } \\
\text { hypoglycemia 2) Identify new } \\
\text { algorithms for insulin dose in closed } \\
\text { loop configuration }\end{array}$ \\
\hline
\end{tabular}

Table 1: Objectives to be achieved to improve performance.
Lack of know how about the time of absorption of insulin, that is currently not less than 15 min $[11,12]$. Currently there are no studies that define the optimal measurement site, but it is known that there are different concentration of glucose in the blood compare to the interstitial fluid [13]. It is well shown the importance of a constant glucose concentration in the blood and prevention of hypoglycemia or hyperglycemia, a result of improper use of insulin pump (CSII). In addition, the insertion of interstitial or intra-cutaneous sensors [14] is often problematic due to poor training, as well as to the currently available intrinsic technological limits of (materials, biological component and hardware) [15-17].

As there is no standardized protocol for communication between devices, each manufacturer adopts its own software, confusing in the user, and limiting the use and interpretation of the data (useful purpose in a proper and continuous monitoring of clinical parameters of diabetic subjects).

The frenetic introduction into the market of new devices by the manufacturers does not allow the development of algorithms able to minimize detection errors of the data $[18,19]$.

The major leaders in the field of biomedical instrumentation for diabetics have proven to be: Abbot, AgaMatrix, Animas, Arkay, Bayer, Bayer, BIONIME, Diabestic Supply of Suncoast, Diagnostic Devices, Dexcom, Lifiscan, Medtronic, Menarini, Nova Biomedical, Roche, Sanofi Aventis. Topic of scientific discussion now turns out to be the finalization of various systems "closed-loop" pointing to the best technological solution that takes into account all the variables that describe the biological system. The main difficulties in the development are related to the difficulties in reproduction of the physiology of insulin and glucose in the data due to the complexity of the human body.

The analyses of recalls showed:

For glucose meters, 59\% of the problems of the devices are data from 'hardware rather than the software (41\%).

$77 \%$ of the insulin pumps considered encountered problems of hardware rather than software $(23 \%)$ and this is a critical issue that could lead to a bad dose of insulin in patients with consequent effects on health.

In $22 \%$ of cases, the user induces problems in the management of the device, because of bad training by the staff, or inadequate interface to the user type.

In addition, the subjectivity of the disease, which is not a negligible element since each patient typically receives a different treatment, affects the therapy by artificial pancreas that cannot be standardized [20-23].

\section{Discussions}

The hospital and industrial research centers should endeavour to improve existing technologies in line with the daily needs of patients. Moreover, it would be important to identify new:

Hardware, customized on the patients target;

Systems that can reduce the problems of interoperability between devices;

Semi-automatic calibrations to reduce possible operating errors [24]. 
Bambino Gesu' Children's Hospital, by the way of great result on a telemedicine case study [25,26], is developing a new Network to help the adolescent patients and their family. By a new App for Android Tablet a Team of Engineers and Doctors are looking to have a standard interface to analyze the diabetic patient data on Telemedicine follow up.

Evaluating the processes of absorption of insulin would facilitate the identification of the more appropriate dosage and reduce the absorption (with inevitable positive impact on the levels of glycosylated hemoglobin), a more appropriate treatment that also means a higher quality of life and reduced direct (health and nonhealth) and indirect costs. The synergy between Clinical, Biomedical Engineers and Computer Services is of great importance to problems resolution and to identify new methods for training for patients and their families [26,27]. The regulations currently in force have not been updated the last five years despite the strong development and innovative technologies.

\section{Conclusion and Future Work}

The study highlighted possible ideas for both technological and scientific improvement, presenting possible new studies to be undertaken, for example about: evaluation of different times depending on the absorption of insertion sites, development for algorithms focus on simplification of business routine and identification for new training techniques for patients and their families on the use of all functions that can be set on different devices. Moreover, critical issues related to the current limits for interoperability between sensors emerged. Insulin pumps and blood glucose meters, should be chosen according to patient's needs, age and personal perceived convenience of use. In addition, the lack of interoperability between devices connected to computers, causes limitations in the choice of software, monitoring and archiving parameters, more 'suitable and intuitive by different users.

\section{References}

1. Danaei G, Finucane MM, Lu Y, Singh GM, Cowan MJ, et al. (2011) National, regional, and global trends in fasting plasma glucose and diabetes prevalence since 1980: systematic analysis of health examination surveys and epidemiological studies with 370 country-years and 2.7 million participants. Lancet 378: 31-40.

2. Jill Weissberg-Benchell, Jeanne Antisdel-Lomaglio, Roopa Seshadri (2012) Insulin Pump Therapy: A meta-analysis. Children's Memorial Hospital, Chicago, Illinois, Giugno.

3. Mariano Agrusta, Marco Tagliaferri (2009) Insulin pumps and surroundings: bioethics in insulin pump therapy to insulina. Seminario Lloyd's Baya hotel.

4. Michael Cook N, Cynthia Girman J, Peter Stein P (2005) Glycemic control continues to deteriorate after sulfonylureas are added to metformin among patients with type 2 diabetes. Diabetes Care American Diabetes Association 28: 995-1000.

5. Ruban Dhaliwal, Ruth Weinstock S (2014) Management of type 1 diabetes in older adults. Diabetes Spectrum 27: 9-20.

6. Joanna Tołwińska, Barbara Głowińska-Olszewska, Artur Bossowski (2013) Insulin Therapy with personal insulin pumps and early angiopathy in children with type 1 diabetes mellitus. Mediators of Inflammation.
7. Bode BW, Schwartz S, Stubbs HA, Block JE (2005) Glycemic characteristics in continuously monitored patients with type 1 and 2 diabetes: normative values. Diabetes Care 28: 2361-2366.

8. Garg SK, Smith J, Beatson C, Lopez-Baca B, Voelmle M, et al. (2009) Comparison of accuracy and safety of the SEVEN and the navigator continuous glucose monitoring systems. Diabetes Technol Ther 11: 65-72.

9. Mary Austin M (2013) Self-monitoring of blood glucose in type 2 diabetes. Diabetes Spectrum 26: 80-81.

10. Bequette BW (2005) A critical assessment of algorithms and challenges in the development of a closed-loop artificial pancreas. Diabetes Technol Ther 7: 28-47.

11. Cobelli C, Renard E, Kovatchev B (2011) Artificial pancreas: past, present, future. Diabetes 60: 2672-2682.

12. American Diabetes Association (2004) Continuous subcutaneous insulin infusion (Position Statement). Diabetes Care 27: S110.

13. Kulku E, Potts RO, Tamada JA, Lesho MJ, Reach G (2003) Phisiological differences between interstitial glucose and blood glucose measured in human subjects. Diabetes Care 26: 2405-2409.

14. Heller A, Feldman B (2008) Electrochemical glucose sensors and their applications in diabetes management. Chem Rev 108: 2482-2505.

15. Jain KK (2007) Applications of nanobiotechnology in clinical diagnostics. Chem 53: 2002-2009.

16. Luo L, Li Q, Xu Y, Ding Y, Wang X, et al. (2010) Amperometric glucose biosensor based on NiFe 2 O 4 nanoparticles and chitosan. Sensors and Actuators B: Chemical 145: 293-298.

17. Wang J (2008) Electrochemical glucose biosensors. Rev 108: 814-825.

18. Yoo EH, Lee SY (2010) Glucose biosensors: an overview of use in clinical practice. Sensors 10: 4558-4576.

19. Klonoff DC (2005) Continuous glucose monitoring: roadmap for 21st century diabetes therapy. Diabetes Care 28: 1231-1239.

20. Kumareswaran K, Evans ML, Hovorka R (2012) Closed-loop insulin delivery: towards improved diabetes care. Discov Med 13: 159-170.

21. Kovatchev BP, Gonder-Frederick LA, Cox DJ, Clarke WL (2004) Evaluating the accuracy of continuous glucose-monitoring sensors: continuous glucose-error grid analysis illustrated by thera sense freestyle navigator data. Diabetes Care Adam 27: 1922-1928.

22. Heller, Ben Feldman (2008) Electrochemical glucose sensors and their applications in diabetes management. Chem Rev 108: 2482-2505.

23. Bruttomesso D, Scotton R, Filippi A, Cipponeri E (2013) Closing the loop in Type 1 Diabetes. G It Diabetol Metab 33: 19-28.

24. Murgia F, Corona B, Bianciardi F, Romano P, Tagliente I, et al. (2014) The application of telemedicine in the follow-up of lung transplantation in a patient with cystic fibrosis. Clin Ter 165: e382-383.

25. Irene Tagliente, Nicola Ullmann, Matteo Ritrovato, Francisco J Leon Trujillo, Riccardo Schiaffini (2015) Benefit of Telemedicine for patients with diabetes mellitus. The Seventh International Conference on eHealth, Telemedicine, and Social Medicine.

26. Schiaffini R, Tagliente I, Carducci C, Ullmann N, Ciampalini P, et al. (2016) Impact of long-term use of eHealth systems in adolescents with type 1 diabetes treated with sensor-augmented pump therapy. J Telemed Telecare 22: 277-281.

27. Taylor A, Wade V, Morris G, Pech J, Rechter S, et al. (2016) Technology support to a telehealth in the home service: Qualitative observations. J Telemed Telecare 22: 296-303. 